\title{
Considerations about the choice of a differintegrator
}

\author{
Manuel D. Ortigueira \\ UNINOVA and DEE ${ }^{1}$ \\ Faculdade de Ciências e Tecnologia \\ da UNL \\ Campus da FCT da UNL, \\ Quinta da Torre, \\ 2829 - 516 Caparica, Portugal \\ mdo@uninova.pt
}

\author{
J. A. Tenreiro Machado \\ Dept. of Electrical Engineering \\ Instituto Superior de Engenharia do \\ Porto \\ R. Dr. Antonio Bernardino de \\ Almeida \\ 4200-072 Porto, Portugal \\ jtm@dee.isep.ipp.pt
}

\author{
J. Sá da Costa \\ Dept. of Mechanical Engineering \\ Instituto Superior Técnico \\ Universidade Técnica de Lisboa \\ Av. Rovisco Pais 1, \\ 1049-001 Lisbon, Portugal \\ sadacosta@dem.ist.utl.pt
}

\begin{abstract}
Despite the great advances in the theory and applications of fractional calculus, some topics remain unclear making difficult its use in a systematic way. This paper studies the fractional differintegration definition problem from a systems point of view. Both local (GrünwaldLetnikov) and global (convolutional) definitions are considered. It is shown that the Cauchy formulation must be adopted since it is coherent with usual practice in signal processing and control applications.
\end{abstract}

\section{INTRODUCTION}

Fractional calculus is a area of mathematics that deals with derivatives and integrals of non integer order (i.e., real or, even, complex) that, often, are joined under the name of differintegration. In the last decade, fractional calculus has been rediscovered by physicists and engineers and applied in an increasing number of fields [1-3], namely in the areas of signal processing, control engineering and electromagnetism [4-10, 18-20]. Despite the developments that have been made, several topics remain without a clear and concise formulation. Surprisingly, one of them is the definition of Fractional Differintegration (FD). In fact, there are several definitions that lead to different results [11-13], making difficult the establishment of a systematic theory of fractional linear systems. In facing this problem, we can assume one of the strategies:

- Elect one formulation, a priori, on the basis of a personal preference;

- Decide to work in a functional space where all the definitions give the same result [14]. Nevertheless, this strategy is interesting only when solving differential equations with inputs in the same space;

- Choose those formulations that assure a generalization of common and useful results or tools.

Bearing these ideas in mind, in this paper we will adopt the third point of view since it is the one that allows building a systematic theory of fractional linear system that resambles the theory of linear (integer order) systems.

The fact of dealing with non-integer order derivatives and integrals constitutes one of the major advantages in using fractional calculus, because solutions are general functions rather than being constrained to the exponential type.
Consequently, we are interested in generalising the useful, and well known results, but there are remarkable differences in this generalization. Integer-order derivatives depend only on the local behaviour of a function, while fractional derivatives depend on the whole history of the function [15]. Therefore, the problem is not just a simple matter of substituting the integer derivative by the fractional derivative; a proper definition of fractional derivative is needed. Moreover, it is important that the adopted definition preserves both the properties of the integer-order differintegration calculus and the fundamental concepts and properties of system theory.

As said previously there are several distinct definitions of $F D$ that are equivalent for a wide class of functions $[1,13]$. Nevertheless, from an engineering point of view most fonnulations reveal compatibility problems with the usual signal processing and systems theory practice. In fact, in signal processing, we often assume that signals have $\Re$ as domain and use the Bilateral Laplace and Fourier Transforms as key tools. Based on these tools, are defined the important concepts of transfer function and frequency response, with properties that we want to preserve in the fractional case. In this line of thought, in this article are considered different differintegration definitions from a common framework and compared in order to establish a practical mathematical tool. In this work, and without loosing generality, we consider two possibilities for the definition of $F D$ :

- An approach based on the generalisation of the usual derivative definition, that is, the GrunwaldLetnikov derivative and integral definitions,

- A global approach based on a convolutional formulation.

As known, any function can be defined in a space isomorphic to a space in which it has been defined in. Thus, it is possible to define the $F D$ through its properties in certain transformed space corresponding to some common transforms like the Laplace Transform (LT). Our starting point is the generalization of the well known property of the $L T$, corresponding to the time domain differentiation:

${ }^{1}$ Also with INESC 


$$
L T\left[D^{\alpha} f(t)\right]=s^{\alpha} F(s), \alpha \in \Re
$$

where $D$ means derivative, $f(t)$ is a signal with (two. sided) Laplace Transform $F(s)\left({ }^{2}\right)$. If $\alpha>0$ we have a fractional derivative, while if $\alpha<0$ is a fractional integral. With this formulation the fractional integral and derivative are mutually inverse operations, which bring an important consequence: the fractional derivative and integral are inverse operations that commute (semigroup property):

$D^{\alpha}\left\{D^{\beta}\right\}=D^{\alpha+\beta}=D^{\beta}\left\{D^{\alpha}\right\}, \alpha, \beta \in \mathfrak{R}$

Unfortunately, this property is not valid in most differintegration definitions [1,13], as it is the so-called Miller-Ross sequential derivative [1].

From a system point of view, we are looking for a "differintegrator" such that its wansfer function is given by $s^{\alpha}$, provided that we have fixed a suitable branch cut line, since it is a multi-valued expression. There are infinite possibilities, but, proceeding as Zavada [16], we choose the negative half-axis. It is clear that if we choose this branch cut line then we force the region of convergence of the $L T$ to be the right $(\operatorname{Re}(s)>0)$ or the left $(\operatorname{Re}(s)<0)$ half plane. This has an important consequence, namely that the differintegrator must be either causal or anti-causal, as in the usual negative integer case, contrarily to the common integer derivatives that are neither causal nor anti-causal (so-called acausal).

In this line of thought, this paper is organized as follows. In sections two and three we discuss two distinct perspectives to differintegration, namely the GrünwaldLetnikov and the convolution approaches, respectively. Based on the previous results section four shows an example common in signal processing and systems theory practice. Finally, section five draws the main conclusions.

\section{GRÜNWALD-LETNIKOV DIFFERINTEGRATION}

\section{A. Derivatives}

Grünwald-Letnikov derivatives are generalisations of the usual derivative definitions. Therefore, $s^{\alpha}(\alpha>0)$ can be considered as the limit when $h \in \mathfrak{R}^{+}$tends to zero in the right hand sides of the following expressions:

$$
\begin{aligned}
& s^{\alpha}=\lim _{h \rightarrow 0+} \frac{\left(1-e^{-s h}\right)^{\alpha}}{h^{\alpha}} \\
& s^{\alpha}=\lim _{h \rightarrow 0^{+}} \frac{\left(e^{s h}-1\right)^{\alpha}}{h^{\alpha}}
\end{aligned}
$$
obtain:

On the other hand, we can use the binomial series to

$$
\begin{aligned}
& \frac{\left(1-e^{-s h}\right)^{\alpha}}{h^{\alpha}}=\frac{1}{h^{\alpha}} \sum_{k=0}^{\infty}(-1)^{k}\left(\begin{array}{l}
\alpha \\
k
\end{array}\right) e^{-s h t}, \operatorname{Re}(s)>0 \\
& \frac{\left(e^{s h}-1\right)^{\alpha}}{h^{\alpha}}=\frac{(-1)^{\alpha}}{h^{\alpha}} \sum_{k=0}^{\infty}(-1)^{k}\left(\begin{array}{l}
\alpha \\
k
\end{array}\right) e^{s h k}, \operatorname{Re}(s)<0
\end{aligned}
$$

In the integer order cases, the right sides in the above expressions are identical. With these formulae, we can write:

\footnotetext{
${ }^{2}$ Exponential order ordinary function or distribution
}

$$
\begin{aligned}
& s^{\alpha}=\lim _{h \rightarrow 0+} \frac{1}{h^{\alpha}} \sum_{k=0}^{\infty}(-1)^{k}\left(\begin{array}{l}
\alpha \\
k
\end{array}\right) e^{-s h k}, \quad \operatorname{Re}(s)>0 \\
& s^{\alpha}=\lim _{h \rightarrow 0+} \frac{(-1)^{\alpha}}{h^{\alpha}} \sum_{k=0}^{\infty}(-1)^{k}\left(\begin{array}{l}
\alpha \\
k
\end{array}\right) e^{s h k}, \quad \operatorname{Re}(s)<0
\end{aligned}
$$

Note the right hand sides regions of convergence. This means that (5a) and (5b) lead to causal and anti-causal derivatives, respectively. These expressions, when inverted to the time domain correspond, respectively, to $\left({ }^{3}\right)$ :

$$
\begin{aligned}
& D_{+}^{\alpha}(t)=\lim _{h \rightarrow 0+} \frac{1}{h^{\alpha}} \sum_{k=0}^{\infty}(-1)^{k}\left(\begin{array}{l}
\alpha \\
k
\end{array}\right) \delta(t-k h) \\
& D_{-}^{\alpha}(t)=\lim _{h \rightarrow 0+} \frac{(-1)^{\alpha}}{h \alpha} \sum_{k=0}^{\infty}(-1)^{k}\left(\begin{array}{l}
\alpha \\
k
\end{array}\right) \delta(t+k h)
\end{aligned}
$$

where $\delta(t)$ is the Dirac delta impulse.

Let $f(t)$ be a limited function and $\alpha>0$. The convolution of (6a) and (6b) with $f(t)$ leads to the Grünwald-Letnikov forward and backward derivatives:

$$
\begin{aligned}
& f_{+}^{(\alpha)}(t)=\lim _{h \rightarrow 0+} \frac{\sum_{k=0}^{\infty}(-1)^{k}\left(\begin{array}{l}
\alpha \\
k
\end{array}\right) f(t-k h)}{h^{\alpha}} \\
& f_{-}^{(\alpha)}(t)=\lim _{h \rightarrow 0+}(-1)^{\alpha} \frac{\sum_{k=0}^{\infty}(-1)^{k}\left(\begin{array}{l}
\alpha \\
k
\end{array}\right) f(t+k h)}{h^{\alpha}}
\end{aligned}
$$

Both expressions agree with the usual derivative definition when $\alpha$ is a positive integer. Moreover, expression (7a) corresponds to the left-hand sided Grünwald-Letnikov fractional derivative while (7b) has the extra factor $(-1)^{\alpha}$, when compared with the right-hand sided Grünwald-Letnikov fractional derivative [13]. Therefore, (7a) and (7b) should be adopted for right and left signals ( ${ }^{4}$, respectively. In [13] it is studied the convergence properties of the above series. It is noteworthy that we can have the forward derivative without existing the backward one and vice-versa. For example, let us apply both definitions to the function $f(t)=$ $e^{a t}$. If $a>0$, expression (7a) converges to $\int_{+}^{(\alpha)}(t)=a^{\alpha} e^{s t}$, while $(7 \mathrm{~b})$ diverges. On the other hand, if $f(t)=e^{-a t}$ equation (7a) diverges while (7b) converges to $f_{-}^{(\alpha)}(t)=$ $(-a)^{a} e^{-a t}$.

Within these definitions, we can apply (7a) or (7b) successively for different values of $\alpha$, leading also to a multi-step derivative $D^{\alpha}=D^{\beta} D^{\gamma} D^{\mu} \ldots D^{\lambda}$, with $\alpha=\beta+\gamma$ $+\beta+\ldots+\lambda$. This means that we have infinite ways of performing a fractional derivative. However, the order in which the fractional differential operators are concatenated is relevant. This is a very important matter that has

\footnotetext{
${ }^{3}$ We do not address here the problem of the convergence of the series \{see [17]\}.

We say that $x(t)$ is a right [left] signal if $\xi(-\infty)-0[x(+\infty)=0]$.
} 
originated a lot of problems mainly when solving fractional differential equations under non zero initial conditions [9]. When $\alpha$ is negative, in general, the series is divergent and an alternative definition needs to be derived as shown in the next section.

\section{B. Integrals}

The expressions for the Grünwald-Letnikov derivatives are not useful for integration [13]. We should expect this because $\frac{h}{1-e^{-s h}} \approx \frac{1}{s}$ is a poor approxirnation and, in fact, the bilinear expression $\frac{h}{2} \frac{1+e^{-s h}}{1-e^{-s h}} \approx \frac{1}{s}$ is superior. Therefore, we can adopt the second approximation to define the fractional integration, leading to a more suitable form for the fractional integral computation. For small $h \in \mathfrak{R}^{+}$:

$\frac{1}{s^{\alpha}} \approx\left(\frac{h}{2} \frac{1+e^{-s h}}{1-e^{-s h}}\right)^{\alpha}=\frac{h^{\alpha}}{2^{\alpha}} \sum_{n=0}^{\infty} C_{n}^{\alpha} e^{-s h n}, \operatorname{Re}(s)>0\left(^{5}\right)$

where

$\mathrm{C}_{n}^{\alpha}=\sum_{k=0}^{n}(-1)^{k}\left(\begin{array}{c}-\alpha \\ k\end{array}\right)\left(\begin{array}{c}\alpha \\ n-k\end{array}\right) \quad n \geq 0$

is the convolution of the coefficients of two binomial series. We can give another form to (9). As

$\left(\begin{array}{l}a \\ k\end{array}\right)=(-1)^{k} \frac{(-a)_{k}}{k !}$

where $(a)_{n}=a(a+1) \ldots(a+n-1)$ is the Pochhammer symbol and remarking that $n !=(-1)^{k}(-n)_{k}(n-k) !$ and $(a)_{n}=$ $(-1)^{k} \cdot(-a-n+1)_{k}(a)_{n-k}$ for $k \leq n$, we obtain:

$\mathrm{C}_{n}^{\alpha}=(-1)^{n} \frac{(-\alpha)_{n}}{n !} \sum_{k=0}^{n} \frac{(\alpha)_{k}(-n)_{k}(-1)^{k}}{(-\alpha-n+1)_{k} k !}$

or

$\mathrm{C}_{n}^{\alpha}=(-1)^{n} \frac{(-\alpha)_{n}}{n !}{ }_{2} \mathrm{~F}_{1}[(\alpha,-n,-\alpha-n+1,-1]$

where ${ }_{2} F_{1}$ is the Gauss Hypergeometric function. Consequently, approximation (8) leads to a GrünwaldLetnikov like fractional integral of order $\alpha$ for a function $f(t)$ :

$$
f^{(a)}(t)=\lim _{h \rightarrow 0+} \frac{h^{\alpha}}{2^{\alpha}} \sum_{n=0}^{\infty} C_{n}^{\alpha} f(t-n h), \alpha<0
$$

For causal signals and $h>0$, the series in (7a) and (13) become finite summations. The formulation (12) is interesting because it allows us to compute $C_{n}^{\alpha}$ recursively. In fact, although the Gauss hypergeometric function does not have a closed form for those arguments, it satisfies the following recursion [6]:

$$
\begin{aligned}
& f(n)=\frac{2 \alpha}{\alpha+n-1} f(n-1)+\frac{(n-1)(n-2)}{(\alpha+n-1)(\alpha+n-2)} f(n-2) \\
& \text { with } f(0)=1 \text {, and } f(1)=2 \text {. } \\
& \text { II. CONVOLUTIONAL DIFFERINTEGRATION }
\end{aligned}
$$

\section{CONVOLUTIONAL DIFFERINTEGRATION}

Here we are going to look for a linear system (the Differintegrator) that has $s^{\alpha}$ - with $\operatorname{Re}(s)>0$ or $\operatorname{Re}(s)<0$ ) -

\footnotetext{
${ }^{5}$ The anti-causal case is similar
}

as Transfer Function. To find its Impulse Response, we look for the inverse Laplace transform of $s^{\alpha}, \delta^{(\alpha)}(t)$, with $\alpha$ $\in \Re$. So the differintegration of a signal $f(t)$ is given by the convolution of $f(t)$ with $\delta^{(\alpha)}(t)$. To present this convolutional differintegration definition, we introduce the following distributions:

$$
\delta_{ \pm}^{(-v)}(t)= \pm \frac{t^{v-1}}{\Gamma(v)} u( \pm t), 0<v<1
$$

$\delta_{ \pm}^{(n)}(t)= \begin{cases} \pm \frac{t^{-n-1}}{\Gamma(v)} u( \pm t) & \text { for } n<0 \\ \delta^{(n)}(t) & \text { for } n \geq 0\end{cases}$

where $n \in Z, \delta^{(\alpha)}(t)$ is the $\alpha$ differintegrator of $\delta(t)$ and $u(t)$ is the Heaviside unit step.

The differintegrations usually used [2] can be classified as right and left sided, respectively:

$f_{r}^{(\alpha)}(t)=[f(t) u(t-a)] * \delta_{+}^{(n)}(t) * \delta_{+}^{(-v)}(t)$

$f_{l}^{(\alpha)}(t)=[f(t) u(b-t)] * \delta_{+}^{(n)}(-t) * \delta_{+}^{(-v)}(-t)$

The orders are given by $\alpha=n-v, n$ being the least integer greater than $\alpha$ and $0<v<1$. In particular, if $\alpha$ is integer then $v=0\left({ }^{6}\right)$. We must remark that, from our point of view, only the cases $a=-\infty$ and $b=+\infty$ cases are acceptable. Otherwise, we are incorporating signal characteristics into a defimition that we think it is not correct. We must state a definition valid for all functions. In other words, the definition must be the same independently of the signal being differintegrated. With this in mind, we rewrite (17a) and (17b) as:

$f_{r}^{(\alpha)}(t)=f(t) * \delta_{+}^{(n)}(t) * \delta_{+}^{(-v)}(t)$
$f_{l}^{(\alpha)}(t)=f(t) * \delta_{+}^{(n)}(-t) * \delta_{+}^{(-v)}(-t)$

The $L T$ of (18a) and (18b) are $s^{\alpha} \mathrm{X}(s)$ and $(-s)^{\alpha} \mathrm{X}(s)$, respectively, that differ on the factor $(-1)^{\alpha}$. This means that it is not a backward differintegration and so it is also unacceptable. From these considerations, we are led to the expressions for the forward and backward differintegrations with general format given by:

$f_{+}^{(\alpha)}(t)=f(t) * \delta_{+}^{(n)}(t) * \delta_{+}^{(-v)}(t)$
$f_{-}^{(\alpha)}(t)=f(t) * \delta_{-}^{(n)}(t) * \delta_{-}^{(-v)}(t)$

With these formulae, integration and derivation are inverse operations. From different orders of commutability and associability in the double convolution we can obtain distinct formulations. For example, in the forward case we have successively the Riemann-Liouville, the Caputo and the Generalised functions differintegration [2]:

$f_{+}^{(\beta)}(t)=\delta_{+}^{(n)}(t) *\left\{f(t) * \delta_{+}^{(-v)}(t)\right\}$
$f_{+}^{(\beta)}(t)=\left\{\mathcal{f}(t) * \delta_{+}^{(n)}(t)\right\} * \delta_{+}^{(-v)}(t)$

\footnotetext{
${ }^{6}$ All the above formulae remain valid in the case of integer integration, provided that we put $\delta^{(0)}(t)=\delta(t)$.
} 
$f_{+}^{(\beta)}(t)=f(t) *\left\{\delta_{+}^{(n)}(t) * \delta_{+}^{(-v)}(t)\right\}$,

where $n \in Z, 0 \leq v<1$. We must remark that (20a) corresponds to a $v$ order integration followed by an $n$ integer order derivative, while in (20b) we have the reverse situation. Concerning equation (20c), the convolution inside brackets is a generalised function given by $[2,18,21]$ :

$\delta_{+}^{(\beta)}(t)=\left\{\delta_{+}^{(n)}(t) * \delta_{+}^{(-v)}(t)\right\}=\frac{t^{-\beta-1}}{\Gamma(-\beta)} u(t)$,

$(\beta=n-v)$ which can be considered as the Impulse Response of the fractional differintegrator. With it we can perform the computation in one step. Moreover, this formulation is a generalization of the well-known Cauchy integral. It is not difficult to obtain the corresponding backward formulations.

\section{SELECTING A DIFFERINTEGRATION}

From previous sections it seems clear that:

- the above three formulations are equivalent when looked from the $L T$ point of view.

- contrarily to the Grünwald-Letnikov differintegration and (20c) in (20a) and (20b) the computation is done in two steps.

We can combine all the differintegrations in the sense that we can decompose the order as $\beta=\beta_{1}+\beta_{2}+\beta_{3}+\ldots+\beta_{n}$ and use anyone to compute the $\beta_{i}(i=1, \ldots, n)$ differintegration. This can lead us to a complicated situation or to results that are far from which we were waiting for. Consider the following problem. We want to check if $x(t)$ is the solution of the differential equation $x^{(3 / 2)}(t)+a x^{(1 / 3)}(t)+b x^{(1 / 5)}(t)=0, a, b \in \Re$, for $t>0$. We can have the options:

a) In the Riemann-Liouville formulation (20a), we have to compute 3 integrals and 4 integer derivatives. In fact, if we want to compute the above derivatives sequentially we have to do the following sequence of computations: $x^{(1 / 5)}(t)=D\left[D^{-4 / 5} x(t)\right] \rightarrow x^{(1 / 3)}(t)=$ $D\left[D^{-13 / 15} x^{(1 / 5)}(t)\right] \rightarrow x^{(3 / 2)}(t)=D\left\{D\left[D^{-5 / 6} x^{(1 / 3)}(t)\right]\right\}$.

b) In the Caputo formulation (20b) we have the same operations but the derivatives and integrations are in reverse order: $x^{(1 / 5)}(t)=D^{-4 / 5}[D x(t)] \rightarrow x^{(1 / 3)}(t)=$ $D^{-13 / 15}\left[D x^{(1 / 5)}(t)\right] \rightarrow x^{(3 / 2)}(t)=D^{-5 / 6}\left\{D\left[D x^{(1 / 3)}(t)\right]\right\}$.

c) In the Cauchy definition (20c) we have 3 fractional derivatives: $x^{(1 / 5)}(t)=D^{1 / 5} x(t) \rightarrow x^{(1 / 3)}(t)=$ $D^{2 / 15}\left[x^{(1 / 5)}(t)\right] \rightarrow x^{(3 / 2)}(t)=D^{7 / 6}\left[x^{(1 / 3)}(t)\right]$.

On the other hand, we must remark that each time we perform an integer order derivative, we are inserting initial conditions that may be meaningless in the problem at hand. In the sequence of operations presented above, we introduce the following initial conditions [1,2,14]:

a) Riemann-Liouville case: $D^{-4 / 5} x(t)_{\mid}+0+,\left.D^{-2 / 3} x(t)\right|_{t=0+}$, $\left.D^{-1 / 2} x(t)\right|_{t=0+}$, and $\left.D^{1 / 2} x(t)\right|_{t=0+}$. To understand these results, we only have to remember that $D[f(t) u(t)]=$ $\left.D f^{(\alpha)}(t)\right] \cdot u(t)+f^{(\alpha)}(0+) \cdot \delta(t)$

b) Caputo case: $\left.x(t)\right|_{t=0},\left.D^{1 / 5} x(t)\right|_{t=0},\left.D^{1 / 3} x(t)\right|_{t=0}$, and $D^{4 / 3} x(t)_{i=0}$. In this case, the fractional integration does not insert an initial condition, contrarily to the integer order derivative. Then, we have $D^{-\alpha}[(t) u(t)]^{\prime}=$ $D^{-\alpha}\left[f^{\prime}(t) \cdot u(t)+f(0+) \cdot \delta(t)\right]$, leading to the result.

c) Cauchy case: $\left.x(t)\right|_{t=0},\left.D^{1,5} x(t)\right|_{t=0}$, and $\left.D^{1 / 3} x(t)\right|_{t=0}$. This result directly from the equation. Of course, we can use other initial conditions by specifying other derivatives, even not "visible" in the equation. We can write, for example: $x^{(3 / 2)}(t)+0 \cdot x^{(1)}(t)+0 x^{(1 / 2)}(t)$ $+a x^{(1 / 3)}(t)+b x^{(1 / 5)}(t)=0$ and insert the corresponding initial conditions [14].

From these considerations we must conclude that Cauchy's is the most useful differintegration, because:

- It does not need superfluous derivative computations

- It does not insert unwanted initial conditions

- It is more flexible and allows a sequential computation.

\section{CONCLUSIONS}

This paper presented two general frameworks for differintegration definitions, namely local and global formulations. The first approach is the Grünwald-Letnikov definition that is a generalisation of the common derivative. It was proposed a new definition for the integral case suitable for numerical algorithms. The global definition has a convolutional format. Among the approaches within this formulation it was choosen the Cauchy defintion because it enjoys all the characteristics required in signal processing and control applications.

\section{REFERENCES}

[1] Miller, K. S. and Ross, B., "An Introduction to the Fractional Calculus and Fractional Differential Equations," John Wiley \& Sons, Inc., 1993.

[2] Podlubny, I., "Fractional Differential Equations," Academic Press, San Diego, 1999.

[3] Westerlund, S., "Dead Matter has Memory", Causal Consulting, Kalmar, Sweden, 2002.

[4] Tenreiro Machado, J. A., "Analysis and Design of Fractional-Order Digital Control Systems", Journal Systems Analysis-Modelling-Simulation, Gordon \& Breach Science Publishers, vol. 27, pp. 107-122, 1997.

[5] Manabe, S., "A Suggestion of Fractional-Order Controller for Flexible Spacecraft Attitude Control", Nonlinear Dynamics, Special Issue on Fractional Order Calculus and Its Applications, vol. 29, Nos. 14, pp. 251-268, July, 2002.

[6] Duarte, F. and Tenreiro Machado J. A., "Chaotic Phenomena and Fractional-Order Dynamics in the Trajectory Control of Redundant Manipulators", "Special Issue on Fractional Order Systems", Joumal of Nonlinear Dynamics, Kluwer, vol. 29, Nos 1-4, pp. 315-342, July 2002.

[7] Moreau, X. , Ramus-Serment, C., and Oustaloup, A., "Fractional Differentiation in Passive Vibration Control", Nonlinear Dynamics, Special Issue on Fractional Order Calculus and Its Applications, vol. 29, n. 1-4, pp. 343-362, July, 2002.

[8] Guijarro N. and Dauphin-Tanguy,G., "Approximation methods to embed the non-integer order models in bond graphs", Signal Processing, special issue on 
Fractional signal processing and applications, vol. 83, no. 11, pp. 2335-2344, Nov. 2003.

[9] Chen Y.Q. and Vinagre, B. M, "A new IIR-type digital fractional order differentiator", Signal Processing, special issue on Fractional Signal Processing and Applications, vol. 83, no. 11, pp. 2359-2365, Nov., 2003.

[10] Leith, J. R., "Fractal scaling of fractional diffusion processes", Signal Processing, special issue on Fractional Signal Processing and Applications, vol. 83, no. 11, pp. 2397-2409, Nov., 2003.

[11] Kalia, R. N. (Ed.), "Recent Advances in Fractional Calculus," Global Publishing Company, 1993.

[12] Nishimoto, K., "Fractional Calculus", Descartes Press Co., Koriyama, 1989.

[13] Samko, S.G., Kilbas, A.A., and Marichev, O.I., "Fractional Integrals and Derivatives - Theory and Applications," Gordon and Breach Science Publishers, 1987.

[14] Ortigueira, M. D., "On the initial conditions in continuous-time fractional linear systems", Signal Processing, Special Issue on Fractional Signal Processing and Applications, vol. 83, no. 11, pp. 2301-2310, Nov. 2003.
[15] Tenreiro Machado, J. A., "A Probabilistic Interpretation of the Fractional-Order Differentiation", Journal of Fractional Calculus \& Applied Analysis, vol. 6, n. 1, pp. 73-80, 2003.

[16] Závada, P., "Operator of Fractional Derivative in the Complex Plane," Communications in Mathematical Physics, 192, pp. 261-285, 1998.

[17] Ferreira, J. C., "Introduction to the Theory of Distributions", Piman Monographs and Surveys in Pure and Applied Mathematics, July 1997.

[18] Ortigueira, M. D., "Introduction to Fractional Signal Processing. Part 1: Continuous-Time Systems", IEE Proc. on Vision, Image and Signal Processing, No.1, pp.62-70, Feb. 2000.

[19] Ortigueira, M. D., "Introduction to Fractional Signal Processing. Part 2: Discrete-Time Systems", IEE Proc. on Vision, Image and Signal Processing, No.1, pp. 71-78, Feb. 2000.

[20] Engheta, N., "Fractional Paradigm in Electromagnetic Theory", in Frontiers in Electromagnetics, D. H. Wemer and R. Mittra (editors), IEEE Press, Chap. 12, November 1999.

[21] Zemanian, A. H., "Distribution Theory and Transform A nalysis," Dover Publications, New York, 1987. 